\title{
Analysis of RDSS Positioning Performance under Elevation Constraints
}

\author{
Yao Fan ${ }^{1}$, Wenxiang Liu ${ }^{1, *}$, Wei Xiao ${ }^{1}$, and Guangfu $\operatorname{Sun}^{1}$ \\ ${ }^{1}$ Engineering Research Center for Position, Navigation and Time, College of Electronic Science, National University of Defense \\ Technology, Deya Road 109, Changsha, China
}

\begin{abstract}
When using RDSS positioning under elevation constraints, traditional positioning accuracy estimation methods may not reflect the impact of satellite ranging error and elevation error on positioning performance accurately. In order to evaluate RDSS positioning performance more accurately, the RDSS positioning principle is presented, and the weighted position dilution of precision (WPDOP) is used to replace the traditional algorithm. The positioning error calculated by WPDOP is closer to actual results, indicating better prediction capability of positioning performance. Under different elevation error conditions, the position dilution of precision (PDOP) distribution of the service area indicates that the difference between the two precision factors is mainly affected by the elevation error and latitude, while the influence of longitude is small.
\end{abstract}

\section{Introduction}

The RDSS positioning and position reporting service is one of the major features of the Beidou global navigation positioning system (BDS-3), and has received more and more attention with the gradual construction of BDS-3. Therefore, the accurate assessment of the positioning performance may facilitate practical use and application development.

After the Beidou-I navigation system completed, the Radio Determination Satellite Service (RDSS) has received intense research.

A detailed summary of various algorithms for Beidou-I navigation and positioning is presented in [1-2]. The traditional precision factor can't be defined because the existence of the elevation error [3]. The actual positioning performance of Beidou-I in the region is obtained by analyzing measured data [4-5]. The position accuracy factor can be defined with reference to the GPS system, but the difference between ranging error and elevation error was ignored [6-7]. The concept of weighted factor is introduced in RNSS [8].

In this paper, the weighted position dilution of precision (WPDOP) algorithm is proposed so that the calculation results of the performance of the RDSS system are closer to reality and the difference between PDOP and WPDOP is analysed.

\section{RDSS positioning principle}

RDSS is a radio positioning system based on the distance measurement, taking the satellite position as a reference point and three-ball rendezvous as the positioning principle. The RDSS positioning signal flow is shown in Figure 1 [9].

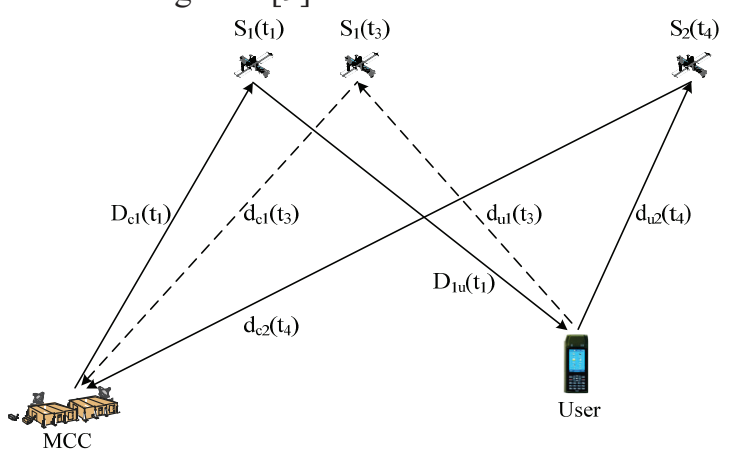

Fig. 1. The RDSS positioning signal flow.

The Master Control Centre (MCC) is the known location on the ground. The satellite $S_{1}$ receives the ranging signal transmitted by MCC at time $t_{1}$. When the user receives any ranging signal transmitted by $S_{1}$ at time $t_{2}$, it transmits an answering signal to each visible satellite. Then the MCC will receive the reply signal returned by each satellite. By measuring the time difference accurately, the sum of the MCC-satellite-user roundtrip distance can be obtained. The user's location requires three visible satellites to calculate theoretically. When only two satellites are available, if the user elevation is known, the two-star positioning will be completed, which is dual-satellite navigation system. In order to make the analysis more representative, the analysis and deduction is proposed under the condition that only two satellites are visible in this paper.

The following ranging equation can be derived as 


$$
\left\{\begin{aligned}
\rho_{1}= & D_{c 1}\left(t_{1}\right)+c \delta t_{c 1 O}+c \delta t_{S 1}\left(t_{1}\right)+D_{1 u}\left(t_{1}\right)+c \delta t_{u}\left(t_{2}\right) \\
& +d_{u 1}\left(t_{3}\right)+c \delta t_{S 1}\left(t_{3}\right)+d_{c 1}\left(t_{3}\right)+c \delta t_{c 1 I}-\varepsilon_{1} \\
\rho_{2}= & D_{c 1}\left(t_{1}\right)+c \delta t_{c 1 O}+c \delta t_{S 1}\left(t_{1}\right)+D_{1 u}\left(t_{1}\right)+c \delta t_{u}\left(t_{2}\right) \\
& +d_{u 2}\left(t_{4}\right)+c \delta t_{S 2}\left(t_{4}\right)+d_{c 2}\left(t_{4}\right)+c \delta t_{c 2 I}-\varepsilon_{2}
\end{aligned}\right.
$$

where $\rho$ is the MCC-satellite-user round trip distance; $\mathrm{t}$ is the arrival time of the signal; Dc1 is the distance between the MCC and the satellite S1; D1u is the distance between the satellite $\mathrm{S} 1$ and the user $\mathrm{u}$; dui and $\mathrm{dci}$ are respectively the distance between user $\mathrm{u}$ and satellite $\mathrm{Si}$ and the distance between satellite $\mathrm{Si}$ and MCC; $\delta$ tc is the equipment delay of the inbound and outbound signal of the central station; $\delta \mathrm{tS}$ is the delay of the satellite repeater's inbound and outbound signal equipment; $\delta$ tu is the delay of the user $u$ forwarding signal; $\varepsilon$ is the pseudo-range measurement error; $\mathrm{c}$ is the speed of light.

In the ECEF coordinate system, the MCC coordinates (xc, yc, zc), the user u coordinate $(\mathrm{x}, \mathrm{y}, \mathrm{z})$, and the satellite position (xSi, ySi, zSi) are set. The user position can be expressed as equation (2) on the premise that the user elevation $h$ is known [1].

$$
x^{2}+y^{2}+\left(z+N_{e} e^{2} \sin B\right)^{2}=\left(N_{e}+h\right)^{2}
$$

where $\mathrm{Ne}$, e, and $\mathrm{B}$ respectively represent the radius of curvature of the reference ellipsoid, the eccentricity of the ellipsoid, and the latitude of the user's location.

The distance between the satellite and the monitoring station Dci and dci can be obtained based on the satellite ephemeris and the location of the monitoring and control station. A corrected pseudo-range observation $\rho$ ' $i$ is defined by eliminating various ranging errors. Corresponding to the observation $\rho$ 'i, the equation becomes

$$
D_{1 u}+d_{c i}=\rho_{i}^{\prime}+\varepsilon_{i}
$$

According to equation (1) (2), considering the threepoint intersection method of elevation constraint, the essence of RDSS positioning algorithm will be to solve the following three-dimensional nonlinear equations as

$$
\left\{\begin{aligned}
f_{1} & =\rho_{1}^{\prime}+\varepsilon_{1} \\
= & \sqrt{\left(x^{\left(S_{1}\right)}-x\right)^{2}+\left(y^{\left(S_{1}\right)}-y\right)^{2}+\left(z^{\left(S_{1}\right)}-z\right)^{2}} \\
& +\sqrt{\left(x^{\left(S_{1}\right)}-x\right)^{2}+\left(y^{\left(S_{1}\right)}-y\right)^{2}+\left(z^{\left(S_{1}\right)}-z\right)^{2}} \\
f_{2} & =\rho_{2}^{\prime}+\varepsilon_{2} \\
= & \sqrt{\left(x^{\left(S_{1}\right)}-x\right)^{2}+\left(y^{\left(S_{1}\right)}-y\right)^{2}+\left(z^{\left(S_{1}\right)}-z\right)^{2}} \\
& +\sqrt{\left(x^{\left(S_{2}\right)}-x\right)^{2}+\left(y^{\left(S_{2}\right)}-y\right)^{2}+\left(z^{\left(S_{2}\right)}-z\right)^{2}} \\
f_{h} & =h+\varepsilon_{h} \\
& =\sqrt{x^{2}+y^{2}+\left(z+N_{e} e^{2} \sin B\right)}-N_{e}
\end{aligned}\right.
$$

Perform the linearization on Equation (4) and use the least-squares method to solve [10]. After completing k-1 iterations, the user position estimate is set to $\boldsymbol{x}_{k-1}=\left(x_{k-1}, y_{k-1}, z_{k-1}\right)$, then

$$
\left[\begin{array}{c}
\Delta x \\
\Delta y \\
\Delta z
\end{array}\right]=\left(\mathbf{G}^{\mathrm{T}} \mathbf{G}\right)^{-1} \mathbf{G}^{\mathrm{T}} \boldsymbol{b},\left[\begin{array}{c}
\varepsilon_{x} \\
\varepsilon_{y} \\
\varepsilon_{z}
\end{array}\right]=\left(\mathbf{G}^{\mathrm{T}} \mathbf{G}\right)^{-1} \mathbf{G}^{\mathrm{T}} \boldsymbol{\varepsilon}
$$

where $\left[\varepsilon_{x}, \varepsilon_{y}, \varepsilon_{z}\right]^{\mathrm{T}}$ is direction component of the error vector $\varepsilon$, and

$$
\mathbf{G}=\left[\begin{array}{ccc}
f_{1 x}^{\prime} & f_{1 y}^{\prime} & f_{1 z}^{\prime} \\
f_{2 x}^{\prime} & f_{2 y}^{\prime} & f_{2 z}^{\prime} \\
f_{h x}^{\prime} & f_{h y}^{\prime} & f_{h z}^{\prime}
\end{array}\right], \boldsymbol{b}=\left[\begin{array}{l}
\rho_{1}^{\prime}-f_{1} \\
\rho_{2}^{\prime}-f_{2} \\
h-f_{h}
\end{array}\right], \boldsymbol{\varepsilon}=\left[\begin{array}{l}
\varepsilon_{1} \\
\varepsilon_{2} \\
\varepsilon_{h}
\end{array}\right]
$$

After obtaining $[\Delta x, \Delta y, \Delta z]^{\mathrm{T}}$, the user position is updated to $\boldsymbol{x}_{k}=\boldsymbol{x}_{k-1}+\Delta \boldsymbol{x}$, and Newton iteration operation is continued as the starting point until the solution accuracy is reached.

\section{Definition of weighted position dilution of precision}

To simplify the analysis, some assumptions are proposed as follows.

The equivalent distance error $\varepsilon i$ of each satellite is normally distributed. Its mean is 0 , and its variance is $\sigma \rho i 2$. The measurement error $\varepsilon$ h of the user's elevation is normally distributed. The mean value is 0 , and the variance is $\sigma \mathrm{h} 2$. The measurement errors between different satellites, satellites and user elevations are not related to each other.

Based on the above assumptions, the covariance matrix of the positioning error can be deduced as

$$
\operatorname{cov}\left(\left[\begin{array}{c}
\varepsilon_{x} \\
\varepsilon_{y} \\
\varepsilon_{z}
\end{array}\right]\right)=\left(\mathbf{G}^{\mathrm{T}} \mathbf{G}\right)^{-1} \mathbf{G}^{\mathrm{T}}\left[\begin{array}{ccc}
\sigma_{\rho 1}^{2} & 0 & 0 \\
0 & \sigma_{\rho 2}^{2} & 0 \\
0 & 0 & \sigma_{h}^{2}
\end{array}\right] \mathbf{G}\left(\mathbf{G}^{\mathrm{T}} \mathbf{G}\right)^{-1}
$$

Defining the weight matrix $\mathrm{W}=\operatorname{diag}(\mathrm{w} 1, \mathrm{w} 2, \mathrm{wh})$, where wi, wh is the weighting factor of the i-th satellite from the error variance and elevation error variance, and wi $=(\sigma \rho i / \sigma U E R E) 2$, wh $=(\sigma \mathrm{h} / \sigma U E R E) 2,(\sigma U E R E) 2$ $=\sigma \mathrm{h} 2+\sigma \rho 12+\sigma \rho 22$.

According to this, the weight coefficient matrix $\mathrm{H}$ can be defined

$$
\mathbf{H}=\left(\mathbf{G}^{\mathrm{T}} \mathbf{G}\right)^{-1} \mathbf{G}^{\mathrm{T}} \mathbf{W} \mathbf{G}\left(\mathbf{G}^{\mathrm{T}} \mathbf{G}\right)^{-1}
$$

Three-dimensional space positioning error standard deviation

$$
\sigma_{P}=W P D O P \cdot \sigma_{U E R E}
$$

Corresponding value of WPDOP

$$
W P D O P=\sqrt{h_{11}+h_{22}+h_{33}}
$$

where hij is the element of the ith row and jth column of matrix H. When $\sigma_{\rho 1}^{2}=\sigma_{\rho 2}^{2}=\sigma_{h}^{2}$, there is $\mathbf{H}=\frac{1}{3}\left(\mathbf{G}^{\mathrm{T}} \mathbf{G}\right)^{-1}$. At this time, the WPDOP is consistent with the traditional definition accuracy factor PDOP. Meanwhile, according to the definition, the greater the difference between the satellite ranging error $\sigma_{\mathrm{P}}$ and the elevation error $\sigma_{h}$, the greater the difference between the WPDOP and the traditional PDOP. 


\section{Assessment of performance under constraints}

\subsection{Analysis of PDOP and WPDOP Positioning Performance Assessment Capabilities}

The location of the $\mathrm{MCC}$ is $\left(116.34^{\circ} \mathrm{E}, 39.98^{\circ} \mathrm{N}, 0 \mathrm{~m}\right)$, the user position is $\left(113.00^{\circ} \mathrm{E}, 28.21^{\circ} \mathrm{N}, 30 \mathrm{~m}\right)$, and the positions of RDSS satellites are $\left(80.20^{\circ} \mathrm{E}, 0.00^{\circ} \mathrm{N}\right.$, $35803000 \mathrm{~m})$ and $\left(140.00^{\circ} \mathrm{E}, 0.00^{\circ} \mathrm{N}, 35807000 \mathrm{~m}\right)$.

Under the different combination of satellite ranging error $\sigma \rho$ and elevation error $\sigma$, the result $\sigma$ Preal of 10000 simulations is approximated as the true positioning error, and the positioning accuracy estimated by the traditional defined PDOP and the newly defined WPDOP is compared and analysed. The difference between the theoretic error variance and the actual result is shown in Table 1.

Table 1. The comparison of PDOP and WPDOP positioning performance evaluation. (unit: $\mathrm{m}$ )

\begin{tabular}{|c|c|c|c|c|}
\hline $\begin{array}{c}\text { Ranging } \\
\text { Error } \\
\sigma_{\rho 1}, \sigma_{\rho 2}\end{array}$ & $\begin{array}{c}\text { Elevation } \\
\text { Error } \sigma_{h}\end{array}$ & $\begin{array}{c}\text { Positioning } \\
\text { Error } \sigma_{\text {Preal }}\end{array}$ & $\begin{array}{c}\sigma_{P_{1}{ }^{*}} \\
\text { (PDOP) }\end{array}$ & $\begin{array}{c}\sigma_{P 2}{ }^{*} \\
\text { (WPDOP) }\end{array}$ \\
\hline \multirow{3}{*}{0} & 0 & 0 & 0 & - \\
\cline { 2 - 5 } & 5 & 9.9086 & 7.1912 & 9.0364 \\
\cline { 2 - 5 } & 10 & 17.9395 & 14.3824 & 18.0727 \\
\cline { 2 - 5 } & 15 & 27.0176 & 21.5736 & 27.1091 \\
\hline \multirow{3}{*}{5} & 0 & 5.4687 & 10.1699 & 8.5723 \\
\cline { 2 - 5 } & 5 & 10.5152 & 12.4555 & 12.4555 \\
\cline { 2 - 5 } & 10 & 18.8573 & 17.6147 & 20.0027 \\
\cline { 2 - 5 } & 15 & 27.5031 & 23.8505 & 28.4321 \\
\hline
\end{tabular}

As can be seen from Table 1 , when $\sigma \rho 1=\sigma \rho 2=\sigma$ h, the positioning error $\sigma$ Preal is 0 , which indicates that the positioning algorithm can accurately solve the user position when the satellite ranging and elevation measurement are accurate. When $\sigma_{\rho 1}=\sigma_{\rho 2}=\sigma_{h}=5, \sigma_{\mathrm{P} 1}$ equals to $\sigma_{\mathrm{P} 2}$, the weight coefficient matrix is a unit matrix, degrading the WPDOP into the traditional definition. Both of them have the same theoretical estimation of the positioning error. In the case of $\sigma_{\rho} \neq \sigma_{h}$, compared with PDOP, the estimated positioning error variance ${ }^{\sigma_{\mathrm{P} 2}}$ calculated by WPDOP is closer to the actual error. That is to say, WPDOP can reflect the system positioning performance more accurately.

\subsection{Differences in PDOP and WPDOP under Different Elevation Error Conditions}

In order to reflect the differences between newly and traditional definitions in different regions, this paper calculates the WPDOP and PDOP for different elevation errors within the system service range. In the area $\left(80^{\circ} \mathrm{E} \sim 140^{\circ} \mathrm{E} ; 10^{\circ} \mathrm{N} \sim 60^{\circ} \mathrm{N}\right)$, the user elevation is set to $0 \mathrm{~m}$, and the sampling point spacing is $1^{\circ}$. The ranging error $\sigma \rho$ is set to $8 \mathrm{~m}$, the height error oh is set to $5 \mathrm{~m}, 8 \mathrm{~m}$, and $15 \mathrm{~m}$ (Nan, Su and Zhou, 2013). The positioning accuracy factor at each point is calculated. The result is shown in Figure 2.

It can be seen from Figure 2 that along the longitude direction, the change of PDOP is slow, but rapidly declining trend from low latitude to high latitude is presented. It is mainly because that the GEO satellite RDSS system used are distributed in the equatorial plane, resulting in the constellation's geometry constraints to the north and south direction is less than the east and west direction.

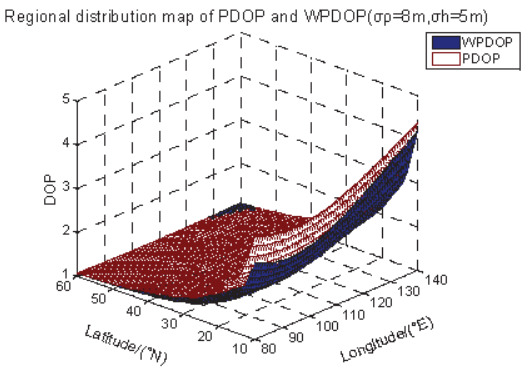

(a) $\sigma_{\rho}=8 \mathrm{~m}, \sigma_{\rho}=5 \mathrm{~m}$

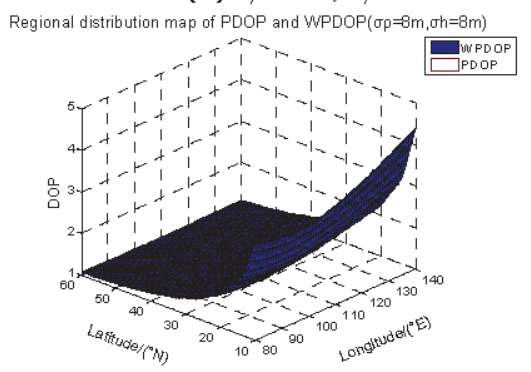

(b) $\sigma_{\rho}=8 \mathrm{~m}, \sigma_{\rho}=8 \mathrm{~m}$

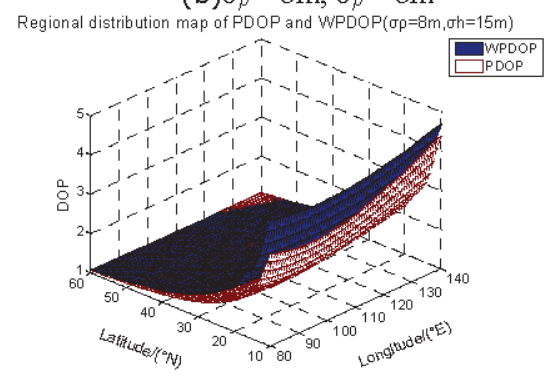

(c) $\sigma_{\rho}=8 \mathrm{~m}, \sigma_{\rho}=15 \mathrm{~m}$

Fig. 2. Regional distribution of PDOP and WPDOP.

The large difference between PDOP and WPDOP in low latitude area, means that the traditional method was used to assess location accuracy will be gravely deviates from the actual performance. Therefore, to assess system service performance accurately, WPDOP should be adopted.

When the elevation error is less than the range error, the elevation has a smaller influence on the positioning performance. But the traditional method will magnifying its influence at this time, leading to the estimation value of the positioning error is higher than the actual situation. When the elevation error is greater than the distance measuring error, elevation impact on positioning performance occupied the main position, which can't be reflected in traditional methods. In this case, traditional 
PDOP will cause the positioning error estimate is lower than actual situation.

\section{Conclusions}

In this paper, the principle of RDSS positioning under the condition of elevation constraint is deduced and the positioning accuracy of RDSS is estimated by WPDOP instead of the traditional PDOP. The simulation results show that the real-time positioning accuracy calculated by WPDOP is more accurate. Moreover, the distribution of PDOP/ WPDOP values in RDSS system service area shows that the value is affected by the latitude significantly instead of the longitude, which is consistent with reality.

This work is supported by the National Science Foundation of China (Nos. 41604016).

\section{References}

1. C.L. Peng, Southwest Jiaotong University (2009).

2. W. Huang, Dalian Maritime University (2012).

3. D.X. Zhang, Radio Engineering (2012).

4. X. Nan, R.R. Su, J.H. Zhou, Science China (2013).

5. R. Guo, R.R. Su, L. Liu, 5th Annual Meeting of China Satellite Navigation Academy (2014).

6. Y.L. Yuan, J. Huang, J. Tao, Computer Applications and Software (2017).

7. Y.L. Yuan, J. Tao, X.Y. Song, International Conference on Information Engineering (2017).

8. M.L. Wang, G.F. Sun, F.X. Wang, Chinese Space Science and Technology, 27 (2007).

9. S.S. Tan, National Defense Industry Press, 61 (2011).

10. G. Xie, Electronic Industry Press, 108 (2009). 